\title{
Human Endogenous Retrovirus K (HERV-K) can drive gene expression as a promoter in Caenorhabditis elegans
}

\author{
Serpen Durnaoglu ${ }^{1,2}$, Heui-Soo Kim ${ }^{3}$, Joohong Ahnn ${ }^{1,2, *} \mathcal{E}$ Sun-Kyung Lee ${ }^{1,2, *}$ \\ ${ }^{1}$ Department of Life Science, ${ }^{2}$ Research Institute for Natural Sciences, Hanyang University, Seoul 04763, ${ }^{3}$ Department of Biological \\ Sciences, College of Natural Sciences, Pusan National University, Busan 46241, Korea
}

\begin{abstract}
Endogenous retroviruses (ERVs) are retrotransposons present in various metazoan genomes and have been implicated in metazoan evolution as well as in nematodes and humans. The long terminal repeat (LTR) retrotransposons contain several regulatory sequences including promoters and enhancers that regulate endogenous gene expression and thereby control organismal development and response to environmental change. ERVs including the LTR retrotransposons constitute $8 \%$ of the human genome and less than $0.6 \%$ of the Caenorhabditis elegans $(C$. elegans) genome, a nematode genetic model system. To investigate the evolutionarily conserved mechanism behind the transcriptional activity of retrotransposons, we generated a transgenic worm model driving green fluorescent protein (GFP) expression using Human endogenous retroviruses (HERV)-K LTR as a promoter. The promoter activity of HERV-K LTR was robust and fluorescence was observed in various tissues throughout the developmental process. Interestingly, persistent GFP expression was specifically detected in the adult vulva muscle. Using deletion constructs, we found that the region from positions 675 to 868 containing the TATA box was necessary for promoter activity driving gene expression in the vulva. Interestingly, we found that the promoter activity of the LTR was dependent on che-1 transcription factor, a sensory neuron driver, and lin-15b, a negative regulator of RNAi and germline gene expression. These results suggest evolutionary conservation of the LTR retrotransposon activity in transcriptional regulation as well as the possibility of che-1 function in non-neuronal tissues. [BMB Reports 2020; 53(10): 521-526]
\end{abstract}

*Corresponding authors. Sun-Kyung Lee, Tel: +82-2-2220-4955; Fax: +82-2-2220-4474; E-mail: sunkyungl@hanyang.ac.kr; Joohong Ahnn, Tel: +82-2-2220-4484; Fax: +82-2-2220-4474; E-mail: joohong@ hanyang.ac.kr

https://doi.org/10.5483/BMBRep.2020.53.10.150

Received 15 July 2020, Revised 5 August 2020, Accepted 20 August 2020

Keywords: Caenorhabditis elegans, che-1, HERV, lin-15b, Retrotransposon

\section{INTRODUCTION}

During evolution, actively replicating retroviruses infected the germline multiple times leading to their transmission into subsequent generations (1). Remnants of viral invasion are present as mobile genetic elements in many metazoan species including mammals and nematodes. Approximately $8 \%$ of the human genome includes HERVs. On the contrary, retrotransposons comprise only $0.6 \%$ of the C. elegans genome (2). Most ERVS are found in truncated form and characterized by multiple stop codons, insertions, and deletions, therefore different sequence variations are detected throughout the genomes of various species (1). These retrotransposons were long-misunderstood to be nonfunctional junk, but are now known to regulate gene expression in various physiological contexts and involved in many pathological conditions including cancers, autoimmune diseases, and neurological disorders, such as lymphomas and melanomas, amyotrophic lateral sclerosis (ALS), and schizophrenia (3). However, the detailed pathophysiological mechanism is largely unknown.

A complete HERV contains an internal region comprising four essential viral genes of gag, pro, pol, and env, flanked by two LTRs arranged in the structure of 5' LTR-gag-pro-pol-env-3' LTR. Most coding sequences in HERVs have been lost by homologous recombination between the two LTRs and remain solitary LTRs (3). Based on the phylogenetic similarity between the pol genes of exogenous members, HERVs are classified into three different groups: class I derived from gammaretrovirus, class II from betaretrovirus, and class III from spumaretrovirus (4). Each class contains many subgroups that are classified based on various analysis strategies. One useful classification depends on the specificity of the human tRNA-primer binding site (PBS) for the reverse transcription process. For example, HERV-W uses tryptophan (W) tRNA whereas HERV-K uses lysine (K) tRNA as a primer (3). The HERV-K clade belongs to Class II betaretrovirus, which is most closely related to mouse mammary tumor virus (MMTV) that causes breast cancer in mice (3). HERV-K contains 11 subgroups, and HML1-11 and HML2 are the most recently integrated and the most active retroviral family, as most of the members still contain the coding region of functional viral proteins and produce retroviral particles (5, $6)$.

ISSN: 1976-670X (electronic edition)

Copyright (c) 2020 by the The Korean Society for Biochemistry and Molecular Biology

(c) This is an open-access article distributed under the terms of the Creative Commons Attribution Non-Commercial License (http://creativecommons.org/licenses/by-nc/4.0) which permits unrestricted non-commercial use, distribution, and reproduction in any medium, provided the original work is properly cited. 
Several studies have shown that HERV-K genes are highly expressed in different types of tumors and cancers $(3,6)$. HERV-K LTRs are active as promoters and regulate the expression of the viral gene and contain regulatory sequences. In transgenic mice harboring HERVs, the rec transcript, generated from the HERV-K env gene by alternative splicing, interferes with germ cell development. A few viable mice developed carcinomas at 19 months of age, which was reminiscent of a precursor lesion preceding a classic human seminoma (7). Transgenic mice expressing the env gene also developed motor dysfunction, reduced volume of the motor cortex, and decreased synaptic activity in pyramidal neurons, all of which are common events in the pathogenesis of ALS, during which HERV-K gene expression plays a pathological role (8). HERV-K119, a humanspecific HERV-K, is highly polymorphic and the pattern of polymorphism differs from other HERV-K elements as it exists as either a full-length HERV-K119 or a solitary LTR, and proviral particles may be generated by reverse transcription $(9,10)$. This genomic architecture of HERV-K119 suggests that it is likely engaged in human physiological functions and pathogenic mechanisms of various diseases.

Functional transcription factor binding sites are commonly predicted within the LTR sequences and some transcription factors may actually bind to the retrotransposons (11). The transactivation response (TAR) DNA-binding protein (TDP-43) specifically binds to pyrimidine-rich motifs in the TAR region, an LTR element in the human immunodeficiency virus type 1 (HIV-1) retrovirus (12). TDP-43 is crucial in the pathology of frontotemporal degeneration (FTD) and ALS and has been shown to regulate HERV-K expression by binding to LTR of HERV-K (13, 14). Furthermore, the expression of human TDP-43 (hTDP-43) in neurons and glia of transgenic Drosophila results in premature death associated with pathological changes in protein aggregation and locomotor impairment (15). TDP-43-induced degeneration results from the activation of the ERV gypsy, which is a retrotransposable element (RTE) structurally related to HERV-K. C. elegans overexpressing hTDP-43 in neurons developed abnormal synapses in motor neurons and exhibited uncoordinated motility (16). Furthermore, knocking out the C. elegans ortholog of hTDP-43 led to the accumulation of transposon-derived RNA transcripts and double-stranded RNA (17). The reported studies strongly suggest that transcriptional regulatory machineries interacting with retrotransposons are conserved in metazoan species, and studying gene regulation by HERV-K in the $\mathrm{C}$. elegans model helps provide some clues to understand the pathophysiological mechanism in various human diseases involving HERV-K.

In this study, we generated a transgenic worm model expressing GFP under the control of the HERV-K119 LTR to investigate differences in temporal and spatial transcriptional activity of the retrotransposon in C. elegans. Interestingly, we found that the LTR was active from the embryonic stage to the adult stage in various tissues, especially in the vulva muscle at the adult stage. The region from position 675 to 868 in the LTR was responsible for the vulva GFP expression. RNAi screening revealed that che-1, a sensory neuron driver, and lin-15b, a negative regulator for RNAi, were required for LTR activation. This study suggests that humans and C. elegans share common transcriptional machinery that activates LTR retrotransposons, and che-1 and lin-15b may be involved in the highly conserved genomic function of retrotransposon regulation.

\section{RESULTS}

\section{HERV-K119 LTR is active as a promoter in C. elegans}

The LTRs retrotransposons can serve as alternative promoters, often conferring some genes with new tissue-specificity and the alternative promoter of LTRs makes it possible to generate different proteins by initiating transcription of different genes (1). Insertion of a solitary LTR can also influence the level of expression of nearby genes (18). The LTRs contain cis-elements which may also recruit various transcription factors including those involved in pathogenic processes.

To investigate the promoter activity of HERV-K in C. elegans, we generated a transgenic worm expressing GFP driven by HERV-K119 LTR. Fluorescence appeared in the embryos even before hatching and expression patterns changed throughout the development process (Fig. 1). Fluorescence was neither ubiquitous nor constitutive and was somewhat varied and distinct in certain tissues and cells. In early embryos and L1 larva, GFP expression was robust in neuronal cells in the head and tail, and the early stages of body wall muscle formation. In L3 to L4 larva, some fluorescence signal was observed in the intestine. In L2 and L3 larva, some neuronal cells were brightly fluorescent throughout the bodies and moderate fluorescence was detected in the intestine. Total GFP expression began diminishing at the L4 stage and finally disappeared in most of the tissues in adults. Interestingly, GFP was expressed almost exclusively in the vulva muscle in adult worms (Fig. $1 \mathrm{E}$ and

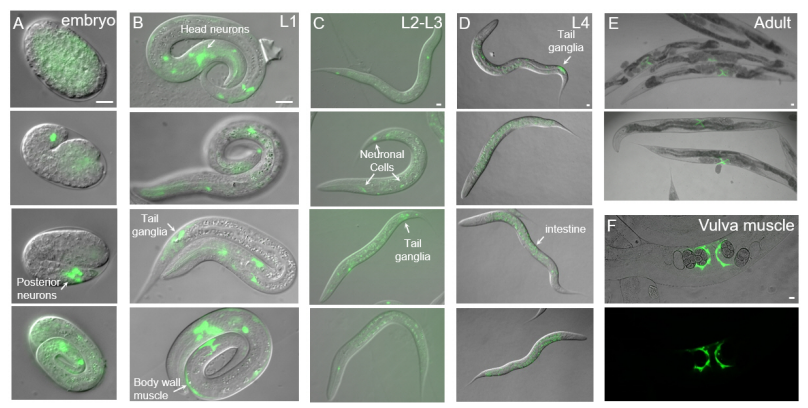

Fig. 1. Promoter activity of HERV-K119 LTR in Caenorhabditis elegans. Merged DIC and fluorescence images are presented. Arrows indicate (A) neuronal precursors in embryos, (B) muscle cells and neurons in the head and tail in early L1 larva, (C) neurons in L2-L3 larva, (D) intestine of L4 larva, and (E) vulva in adults. (F) Selective GFP expression in the vulva muscles of adult worms. Scale bars, $10 \mu \mathrm{m}$. 
1F). These data suggest that HERV-K119 LTR function as a promoter and its regulatory elements should be compatible with activation of $C$. elegans transcription factors.

\section{HERV-K119 LTR regions are specific for gene expression in C. elegans}

To determine the region required for the GFP expression observed in transgenic worms with the full-length HERV-K119 LTR, we constructed plasmids harboring four different deletions in the HERV-K119 LTR sequence and injected them into N2 wild-type worms to generate transgenic worms (Fig. 2A). Among the different deletions, only the deletion- 4 construct resulted in complete loss of GFP expression, while the others exhibited a similar degree of fluorescence as the full-length construct worms (Fig. 2B). This indicated that the 194-bp fragment at position 675 to 868 was essential for LTR promoter activity. We further analyzed the regulatory feature of HERV-K119 LTR

(A)

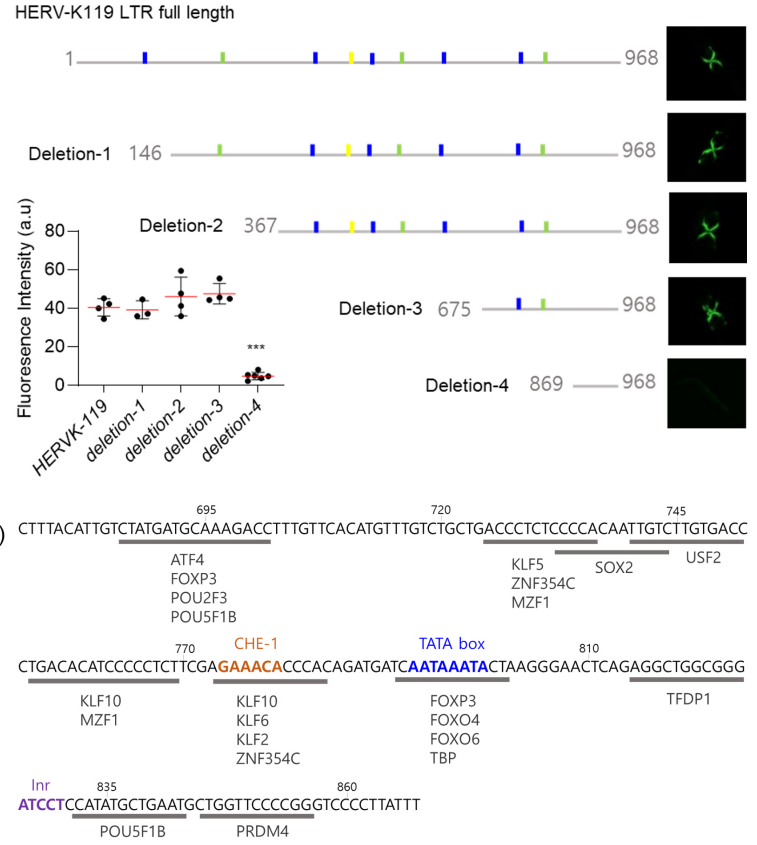

Fig. 2. Promoter function of HERV-K119 LTR. (A) Schematic representations of the HERV-K119 LTR deletions are presented as horizontal gray lines with the corresponding representative green fluorescence images of each transgenic animal. Deletion 4 results in a complete loss of GFP fluorescence. GPminer (http://gpminer.mbc.nctu.edu.tw/) prediction of regulatory motifs is depicted as short vertical lines. Blue, green, and yellow indicate TATA boxes, GC-boxes, and the strong CCAAT box, respectively. (B) Quantification of fluorescence intensities in vulva muscles of transgenic worms. ${ }^{*} * * P<0.001$. (C) Regulatory elements in the 194-bp fragment from position 675 to 868 in the HERV-K119 LTR, predicted by GPminer and JASPAR (http://jaspar. genereg.net/). The TATA box and Inr as well as different transcription factor binding sites are indicated. Human transcription factor binding sites are underlined in gray and $\mathrm{CHE}-1$ is a $\mathrm{C} 2 \mathrm{H} 2$ zincfinger transcription factor of Caenorhabditis elegans. utilizing a bioinformatics approach. Gene Promoter Minor (GPminer, http://gpminer.mbc.nctu.edu.tw/) identified a potential TATA box at the 793 bp position and a transcription initiation-like sequence (Inr) at the 826 bp position. These sequences were previously shown to play an essential role in the transcriptional regulatory function of HERV-K LTR (Fig. 2A). Furthermore, the JASPAR database (http://jaspar.genereg.net/) indicated that the region contained many motifs that were predicted to bind to various human and C. elegans transcription factors (Fig. 2C and Supplementary Table 1). Comprehensively, these results strongly suggest that the HERV-K LTR serves as an active transcriptional regulatory element such as a promoter/ enhancer unit in C. elegans.

\section{C. elegans transcription factors and the promoter activity of HERV-K119 LTR}

We next attempted to determine whether $C$. elegans transcription factors are involved in GFP expression driven by HERV-K119 LTR. We used RNAi to target transcription factors predicted by the JASPAR database, as well as lin-11, lin-15B, lin-13, and lin-39 that regulate the development of vulva muscle (www.wormbase. org). The GFP intensity was significantly reduced in worms treated with RNAi targeting che-1 but increased in those with lin-15b RNAi (Fig. 3A and 3B).

\section{che-1 and lin-15b affect the promoter activity of HERV-K119 LTR in the vulva of $C$. elegans}

To further investigate whether che-1 and lin-15b are essential for HERV-K119 LTR transcriptional activity, we injected a construct of HERV-K119 LTR:GFP into che-1(p696) and lin-15b(n744) loss-of-function mutant worms. Fluorescence in the che-1(p696); HERV-K119:GFP worms was significantly reduced compared to wild-type control worms, recapitulating the result of the che-1 RNAi experiment (Fig. 3C and 3D). However, a dramatic reduction in GFP intensity was observed in lin-15b;HERV-K119: GFP worms, which contradicted the increased GFP expression observed following lin-15b RNAi (Fig. 3C and 3D).

We found that there were very few lin-15B; HERV-K119:GFP worms with high expression of GFP in their vulva muscles (Fig. 3C-iv). Even though those worms had eggs in their uterus, they did not lay eggs and sometimes had worm-bagging. Eggs with high expression of GFP were infrequently observed and did not hatch. (Fig. 3C-v). Only worms that expressed GFP at low levels were able to lay eggs that were hatched to release larvae. It is hypothesized that overexpression of HERV-K119: GFP in lin-15B(n744) mutants may have interfered with the normal physiology of the worms thereby leading to embryonic lethality with a possible association with enhanced RNAi in $n 744$ allele mutants, although egg-laying activities of lin-15B(n744) mutant worms were not significantly different from the wild type (Supplementary Fig. 1). Altogether, it is proposed that HERV-K119 LTR can act as a promoter driving gene expression in C. elegans and che-1 and lin-15b may in turn be involved in transcriptional regulation of HERV-K119 LTR. 


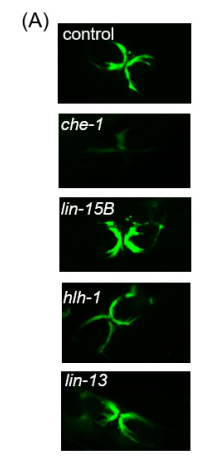

(C)

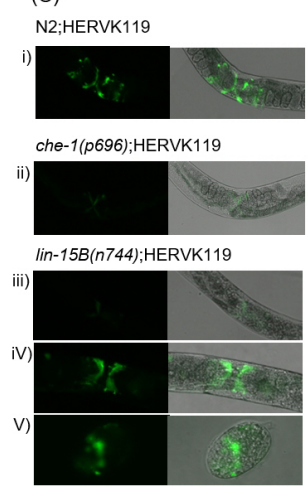

(B)

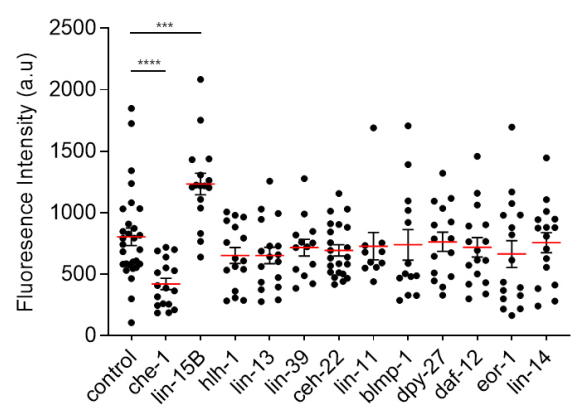

(D)

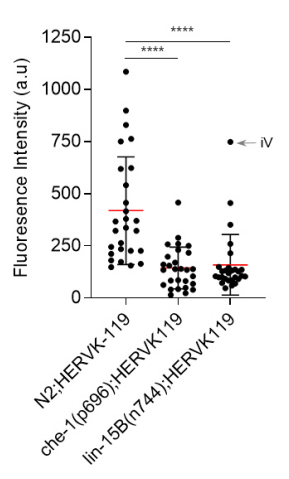

Fig. 3. RNAi targeting Caenorhabditis elegans transcription factors. (A) Representative fluorescence images of vulva muscles in worms treated with RNAi. (B) Quantification of GFP intensities (t-test. ***P $<0.001$, $* * * * \mathrm{P}<0.0001)$. Green fluorescent protein expression is driven by HERV-K119 LTR in che-1(p696) and lin-15B(n744) mutants. (C) Images show expression of green fluorescent protein driven by HERV-K119 LTR in wild-type (C-i), che-1(p696) mutants (C-ii), and lin-15B(n744) mutants (C-iii). In lin-15B(n744) background, while most of the worms exhibit low GFP expression, very few worms expressing a high level of green fluorescent protein do not lay eggs (C-iv), and some eggs with high fluorescence do not hatch, either (C-v). (D) Green fluorescent protein levels in day-1 old transgenic worms were quantified (***P $<0.001, * * * * P<0.0001$, Student's t-test).

\section{DISCUSSION}

ERVs and retrotransposons play a critical role in gene expression and thereby participate in various biological processes in organismal development, physiology, and pathology. The integration of various polymorphic forms of ERVs and retrotransposons into genomes of various metazoan species indicates the subsequent evolution of various mobile elements in different species. Although the genomic structure and regulation of ERVs and transposons are species-specific, some still share common molecular biological mechanisms that regulate gene expression, as shown in flies or worms overexpressing hTDP-43 or with the knock-down of a TDP-43 homolog. In this study, we found that HERV-K LTR can drive gene expression in C. elegans and that this activity was affected by CHE-1 and LIN-15b transcription factors.

The C. elegans genome contains no fewer than 19 families or 37 clusters of ERVs and LTR retrotransposons, including at least 58 full-length ERVs and 297 single $\operatorname{LTR}(19,20)$. A phylogenetic analysis based on the amino acid sequence of reverse transcriptase indicated that Cer1-Cer6 retrotransposons fall in the same family domain as the gypsy/Ty3 groups that are adjacent to the family domain that includes HERV-K. Although most of the retrotransposons are silenced by ADARs (adenosine deaminases acting on double-stranded RNA) and nuclear RNAi to protect endogenous genome structure, a few C. elegans retrotransposons have been reported to be actively expressed $(21,22)$. Cer 1 produces capsids that assemble viruslike particles (VLPs) in C. elegans germ cells (23). The formation of VLPs in C. elegans germ cells is dynamically regulated and is dependent on temperature and age. We observed both temporal and spatial changes in the pattern of GFP expression in worms harboring HERV-K119 LTR:GFP during development. The dynamic promoter activity of human retrotransposons in C. elegans indicates that human retrotransposons may be competent for the regulatory control of worm retrotransposons depending on the physiological and environmental status of the worms (24).

Several studies have revealed the activation of HERV-K LTR by transcription factors in diseases such as cancers and neurological disorders. For example, TDP-43 interacts with HERV-K LTR, thereby contributing to the pathogenesis of FTD and ALS (13). Bioinformatics tools indicate that HERV-K119 includes sequence motifs such as TATA boxes, GC-boxes, and CCAAT boxes, as well as various $C$. elegans transcription factor binding motifs (Fig. 2 and Supplementary Table 1). The promoter activity of HERV-K in C. elegans was significantly reduced following che-1 RNAi or mutation of che-1. Interestingly, the predicted che-1 binding site is located directly upstream of the TATA box and Inr in the 194 bp active promoter region and overlapped with other predicted human transcription factor binding motifs. che-1 is known as a terminal regulator for ASE sensory neurons in C. elegans, and various homologs of che-1 can be detected in human, murine, and fly genomes using a BLAST search (Fig. $4 \mathrm{~A}$ and Supplementary Table 1). The zinc-finger domain of che-1 is identical to that of GLASS, and human ZNF500 shares the highest homology with the domain (25). Altogether, these data suggest that che- 1 may actively participate in the promoter activity of the 194 bp-region of HERV-K119 LTR and may function in retrotransposon transcription in worms.

In this study, the promoter activity of HERV-K119 was increased by RNAi targeting lin-15b but significantly decreased in lin-15b mutant worms (Fig. 2, 3). lin-15b is a class B synthetic multivulva gene that functions in the signaling pathway to regulate vulva formation in parallel to class $A$ genes (26). Lin-15B protein contains a putative THAP zinc-finger domain that is found in transposases, and an RNaseH-like superfamily domain that is present in the reverse transcriptase of retroviruses (Fig. 4) (27-30). In the absence of lin-15b, RNAi 
(A)

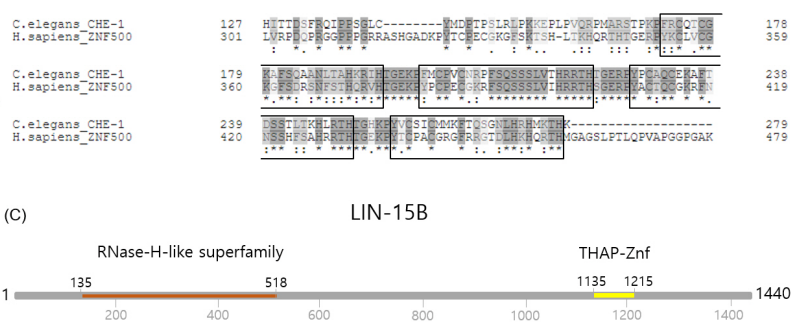

Fig. 4. Phylogenetic relationships and conserved regions of $\mathrm{CHE}-1$. (A) The phylogenetic tree was constructed by MEGA X (Kumar et al. 2018) with nine transcription factors. The neighbor-joining method was used with 500 bootstrap replications. Bootstrap percentages are shown next to the branches. Evolutionary distances were computed using the p-distance method and are provided in the unit number of amino acid differences per site. There were a total of 639 positions in the final dataset. (B) The amino acid alignment of $C$. elegans CHE-1 and human ZNF500 protein. Zinc-finger domains are shown in boxes. (C) Domain structure of LIN-15B by InterPro (Mitchell et al., 2018). LIN-15B consists of 1440 AA residues. The ribonuclease H-like (RNHL) superfamily domain (residues, 135-518) and the THAPtype zinc-finger domain (THAP-Znf, residues, 1135-1215) are indicated.

efficiency is generally increased due to the somatic misexpression of $\mathrm{P}$ granules, which silences LTR retrotransposons and other transgenes $(21,31-33)$. lin-15b drives the repressive histone demethylation of histone $\mathrm{H} 3$ on lysine 9 ( $\mathrm{H} 3 \mathrm{~K} 9 \mathrm{me} 2)$ in the promoter region of germline genes and other synMuv B gene targets to silence their expression (34). Thus, both enhanced RNAi and expression of P granule components in lin-15b mutants may suppress transgene expression in vulva driven by HERV-K119 LTR. Interestingly, lin-15b transcripts are enriched in ASER neurons, in which che-1 is known to be a terminal regulator (www.wormbase.org), and suggests a novel regulatory mechanism that is functionally associated with ASE sensory neurons and the vulva.

In conclusion, this study shows that HERV-K LTR can drive gene expression in C. elegans and che-1 and lin-15b are required for the promoter activity of human LTRs. The results suggest a novel function of che-1 in non-neuronal tissues as well as retrotransposon regulator activity mediated by lin- $15 b$ in C. elegans. This study also suggests a novel pathway associating ASE taste neurons with the functional integrity of the vulva. HERV-K LTRs play a critical role in mediating the pathological process of human cancers and neurodegenerative diseases. It is hypothesized that further studies on understanding the suppression of HERV-K promoter activities by che- 1 and lin-15b using $C$. elegans would help to understand the mecha- nism through which endogenous retroviruses control internal and external gene expression that is critical for disease onset and progression, as well as organismal adaptation and susceptibility to retroviruses.

\section{MATERIALS AND METHODS}

\section{Strains, maintenance, and RNAi}

Worms were maintained according to the standard protocol (Brenner, 1974) at $20^{\circ} \mathrm{C}$. The N2 Bristol strain, che-1 (p696) and lin-15B (n744) were obtained from the Caenorhabditis Genetics Center (CGC). The feeding RNAi was performed as described previously (Kamath et al., 2003).

\section{Constructs and transgenic worms}

HERV-K119 LTR and various deletion mutant forms were cloned into the pPD95.77 vector. To construct the eor-1 RNAi clone, the coding region was amplified from cDNA prepared from worm extracts and then cloned into the L4440 empty vector (Kamath et al., 2003). Primers used in this study are listed in Supplementary Table 2. Transgenic strains were generated by the standard microinjection technique (Mello et al., 1991).

\section{Fluorescence microscopy}

After RNAi treatment, one-day-old adult worms were selected and images were taken using a 20X objective with the Zeiss AxioCam microscope. Fluorescence intensity was quantified using the Axio Cam software (AxioVs40 V4.8.2.0). The Student's t-test was performed to determine statistical significance.

\section{ACKNOWLEDGEMENTS}

This research was supported by the National Research Foundation of Korea (NRF) funded by the Ministry of Education, Science, and Technology (NRF-2018R1A2A3074987 to S-K.L.; NRF-2018 R1D1A1B07046893 and NRF-2015S1A3A2046684 to J.A.).

\section{CONFLICTS OF INTEREST}

The authors have no conflicting interests.

\section{REFERENCES}

1. Craig NL, Craigie $R$, Gellert $M$ and Lambowitz Alan $M$ (2015) Mobile DNA III, ASM Press, Washington, D.C. 1051-1078

2. Bessereau JL (2006) Transposons in C. elegans; in, The C. elegans Research Community, WormBook, doi/10.1895/ wormbook.1.70.1

3. Subramanian RP, Wildschutte JH, Russo C and Coffin JM (2011) Identification, characterization, and comparative genomic distribution of the HERV-K (HML-2) group of human endogenous retroviruses. Retrovirology 8, 90

4. Vargiu L, Rodriguez-Tomé P, Sperber GO et al (2016) 
Classification and characterization of human endogenous retroviruses; mosaic forms are common. Retrovirology 13, 7

5. Kim HS (2012) Genomic impact, chromosomal distribution and transcriptional regulation of HERV elements. Mol Cells 33, 539-544

6. Garcia-Montojo M, Doucet-O'Hare T, Henderson $L$ and Nath A (2018) Human endogenous retrovirus-K (HML-2): a comprehensive review. Crit Rev Microbiol 44, 715-738

7. Galli UM, Sauter M, Lecher B et al (2005) Human endogenous retrovirus rec interferes with germ cell development in mice and may cause carcinoma in situ, the predecessor lesion of germ cell tumors. Oncogene 24, 3223-3228

8. Li M, Radvanyi L, Yin B et al (2017) Downregulation of human endogenous retrovirus type $K$ (HERV-K) viral env RNA in pancreatic cancer cells decreases cell proliferation and tumor growth. Clin Cancer Res 23, 5892-5911

9. Shin W, Lee J, Son S-Y, Ahn K, Kim H-S and Han K (2013) Human-specific HERV-K insertion causes genomic variations in the human genome. PloS One 8, e60605

10. Lee W-C, Kim D-Y, Kim M-J et al (2019) Delay of cell growth and loss of stemness by inhibition of reverse transcription in human mesenchymal stem cells derived from dental tissue. Anim Cells Syst (Seoul) 23, 335-345

11. Bourque G, Leong B, Vega VB et al (2008) Evolution of the mammalian transcription factor binding repertoire via transposable elements. Genome Res 18, 1752-1762

12. Ou SH, Wu F, Harrich D, García-Martínez LF and Gaynor RB (1995) Cloning and characterization of a novel cellular protein, TDP-43, that binds to human immunodeficiency virus type 1 TAR DNA sequence motifs. J Virol 69, 3584-3596

13. Li W, Lee $M H$, Henderson L et al (2015) Human endogenous retrovirus-K contributes to motor neuron disease. Sci Transl Med 7, 307ra153

14. Mitra J, Guerrero EN, Hegde PM et al (2019) Motor neuron disease-associated loss of nuclear TDP-43 is linked to DNA double-strand break repair defects. Proc Natl Acad Sci U S A 116, 4696-4705

15. Krug L, Chatterjee N, Borges-Monroy R et al (2017) Retrotransposon activation contributes to neurodegeneration in a Drosophila TDP-43 model of ALS. PLoS Genet 13, e1006635

16. Ash PEA, Zhang YJ, Roberts CM et al (2010) Neurotoxic effects of TDP-43 overexpression in C. elegans. Hum Mol Genet 19, 3206-3218

17. Saldi TK, Ash PEA, Wilson G et al (2014) TDP-1, the Caenorhabditis elegans ortholog of TDP-43, limits the accumulation of double-stranded RNA. EMBO J 33, 29472966

18. Macfarlan TS, Gifford WD, Agarwal S et al (2011) Endogenous retroviruses and neighboring genes are coordinately repressed by LSD1/KDM1A. Genes Dev 25, 594-607

19. Ganko EW, Fielman KT and McDonald JF (2001) Evolutionary history of Cer elements and their impact on the C. elegans genome. Genome Res 11, 2066-2074

20. Rho M, Choi J-H, Kim S, Lynch M and Tang H (2007) De novo identification of LTR retrotransposons in eukaryotic genomes. BMC genomics 8, 90

21. Fischer SEJ and Ruvkun G (2020) Caenorhabditis elegans ADAR editing and the ERI-6/7/ MOV10 RNAi pathway silence endogenous viral elements and LTR retrotransposons. Proc Natl Acad Sci U S A 117, 5987-5996

22. Ni JZ, Kalinava N, Mendoza SG and Gu SG (2018) The spatial and temporal dynamics of nuclear RNAi-targeted retrotransposon transcripts in Caenorhabditis elegans. Development 145, dev167346

23. Dennis S, Sheth U, Feldman JL, English KA and Priess JR (2012) C. elegans germ cells show temperature and agedependent expression of Cer1, a Gypsy/Ty3-related retrotransposon.PLoS Pathog 8, e1002591

24. Kwon S, Kim EJE, Lee SJV (2018) Mitochondria-mediated defense mechanisms against pathogens in Caenorhabditis elegans. BMB Rep 51, 274-279

25. Etchberger JF, Lorch A, Sleumer MC et al (2007) The molecular signature and cis-regulatory architecture of a C. elegans gustatory neuron. Genes Dev 21, 1653-1674

26. Boxem M and van den Heuvel S (2002) C. elegans class B synthetic multivulva genes act in $\mathrm{G}(1)$ regulation. Curr Biol 12, 906-911

27. Clouaire $T$, Roussigne $M$, Ecochard V, Mathe $C$, Amalric $F$ and Girard J-P (2005) The THAP domain of THAP1 is a large $\mathrm{C} 2 \mathrm{CH}$ module with zinc-dependent sequence-specific DNA-binding activity. Proc Natl Acad Sci U S A 102, 69076912

28. Roussigne $M$, Kossida S, Lavigne A-C et al (2003) The THAP domain: a novel protein motif with similarity to the DNA-binding domain of $\mathrm{P}$ element transposase. Trends Biochem Sci 28, 66-69

29. Majorek KA, Dunin-Horkawicz S, Steczkiewicz K et al (2014) The RNase H-like superfamily: new members, comparative structural analysis and evolutionary classification. Nucleic Acids Res 42, 4160-4179

30. Moelling K, Broecker F, Russo G and Sunagawa S (2017) RNase $\mathrm{H}$ As Gene Modifier, Driver of Evolution and Antiviral Defense. Front Microbiol 8, 1745-1745

31. Lehner B, Calixto A, Crombie C et al (2006) Loss of LIN-35, the Caenorhabditis elegans ortholog of the tumor suppressor p105Rb, results in enhanced RNA interference. Genome Biol 7, R4

32. Wang D, Kennedy S, Conte D et al (2005) Somatic misexpression of germline $P$ granules and enhanced RNA interference in retinoblastoma pathway mutants. Nature 436, 593-597

33. Wu X, Shi Z, Cui M, Han M and Ruvkun G (2012) Repression of Germline RNAi Pathways in Somatic Cells by Retinoblastoma Pathway Chromatin Complexes. PLoS Genet 8, e1002542

34. Rechtsteiner A, Costello ME, Egelhofer TA, Garrigues JM, Strome S and Petrella LN (2019) Repression of Germline Genes in Caenorhabditis elegans Somatic Tissues by H3K9 Dimethylation of Their Promoters. Genetics 212, 125-140 